\title{
Arrhythmic manifestation of non-compaction cardiomyopathy of both ventricles
}

\author{
Paulina Dubiel, Grzegorz Jan Kamiński (iD,Andrzej Curzytek
}

Department of Cardiology, SP ZOZ MSWiA, Rzeszów, Poland

\section{Abstract}

This paper presents a case report of a patient diagnosed with a rare non-compaction cardiomyopathy of both ventricles. The diagnosis was based on echocardiography after excluding other possible causes of myocardial damage. The reason for the initiation of cardiological diagnostics was a history of syncope, the cause of which was finally considered to be paroxysmal ventricular tachycardia. In the secondary prevention of sudden cardiac death, the patient was implanted with a cardioverter-defibrillator. The thrombus found in the left ventricle was successfully treated with anticoagulants.

Key words: non-compaction cardiomyopathy, heart failure, arrhythmic manifestation, echocardiography, ventricular tachycardia, implantable cardioverter-defibrillator, left ventricular apical thrombus

Folia Cardiologica 2021; 16, 2: 134-137

\section{Introduction}

Non-compaction cardiomyopathy (NCC) is a rare form of cardiomyopathy, defined by the European Society of Cardiology (ESC) classification as unclassified cardiomyopathy. On the other hand, the American Heart Association (AHA) classification consideres NCC to be genetic cardiomyopathy. NCC most commonly affects the left ventricle and manifests with abnormal myocardial structure. This pathology involves the presence of a two-layered left ventricular wall within the non-compacted segments: an inner non-compacted (NC) layer and an compacted (C) layer [1, 2].

The prevalence of NCC is not precisely known. According to various sources it ranges from $0.05-0.26 \%$ of the general population of adults referred to echocardiography. Clinically, NCC may be asymptomatic for a long time. The first symptoms are those typical of heart failure or cardiac arrhythmia. Sudden cardiac death may be the first manifestation. The triad of typical symptoms is completed by thromboembolic complications. In this group of patients, monomorphic ventricular tachycardia predominates. Both, monomorphic and polymorphic, ventricular tachycardia can be found in one third of patients. The diagnosis is based on transthoracic echocardiography (TTE) and cardiac magnetic resonance (CMR).

The most clinically useful criteria for diagnosing left ventricular non-compaction (LVNC) are those presented by Jenni [3]. The diagnosis of this cardiac pathology requires the detection of a two-layered structure of the left ventricular wall together with an outer, epicardial, compacted layer (C) and an inner, endocardial, thicker non-compacted layer (NC); a thickness ratio of NC:C > 2 measured in parasternal short axis view during late systole, and the presence of blood flow directly from the left ventricular chamber into the sinuses formed between trabeculae of the non-compacted layer. Right ventricular non-compaction (RVNC) may accompany LVNC, and there are no separate, generally accepted criteria for diagnosing this pathology in the right ventricle. Currently, there is no specific therapy for this condition, and a medical procedure should depend on clinical picture. In this group of patients, routine anticoagulant therapy remains to be discussed. 


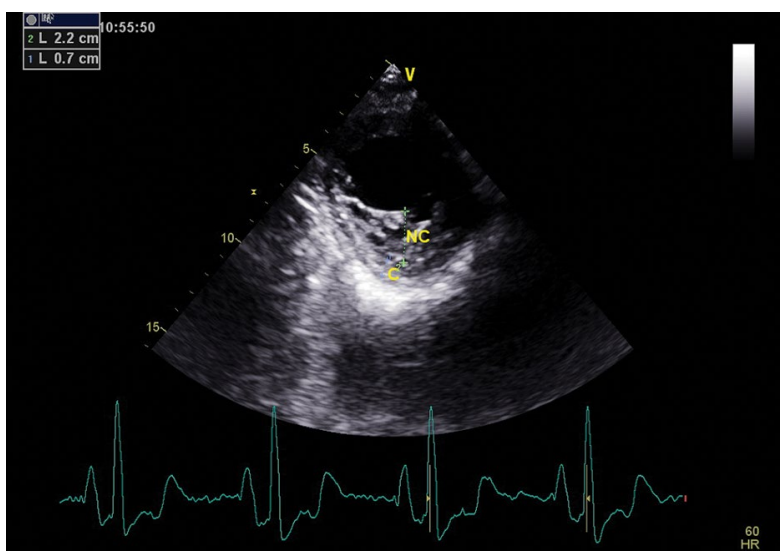

Figure 1. The ratio between non-compacted (NC) endocardial layer and compacted $(\mathrm{C})$ epicardial layer in the end-systolic phase and parasternal short axis view

\section{Case study}

A man, aged 54, was admitted to the cardiology department for extended diagnosis due to a complete and brief loss of consciousness while driving a car. It was the second loss of consciousness in the patient's life.

Based on the patient's history, it was found that he had good health and for approx. a year he reported no complaints, excluding brief and mild dizziness. The possibility of toxic myocardial injury was excluded. The patient's family history raised the suspicion of sudden cardiac death in his father, who died at a young age. There were no overt signs of heart failure in the physical examination.

Electrocardiogram (ECG) monitoring showed sinus rhythm with QRS complex frequency of approx. 60/min., cardiac axis - normogram, negative T-waves in II, III, aVF, V5, V6. The poor R-wave progression in V1-V4 also paid particular attention. The ECG monitoring was stable during hospitalisation. No significant abnormalities were found on chest X-ray. In laboratory studies, an unremarkable increase in the $\mathrm{N}$-terminal pro-B-type natriuretic peptide (NT-proBNP) was detected $-867.60 \mathrm{pg} / \mathrm{mL}$ [norm (N): 0-125]. Normal calcium, ferritin, iron, total iron binding capacity (TIBC) and creatine kinase values were also found. D-dimer levels were normal: $<170 \mathrm{ng} / \mathrm{mL}$ ( $\mathrm{N}:$ 0-500). Echocardiography found left ventricular chamber dilatation [left ventricular end-diastolic diameter) (LVEDD) $=70 \mathrm{~mm}$, left ventricular end-systolic diameter (LVEDS) $=63 \mathrm{~mm}$ ] with normal thickness of the basal segments of the interventricular septum and inferior wall, as well as generalised left ventricular hypokinesis with a biplane left ventricular ejection fraction (LVEF) of $22 \%$. In the left ventricular apex and within the apical and mid segments of the inferior and lateral walls, as well as within the apical segment of the anterior wall, increased trabeculation was

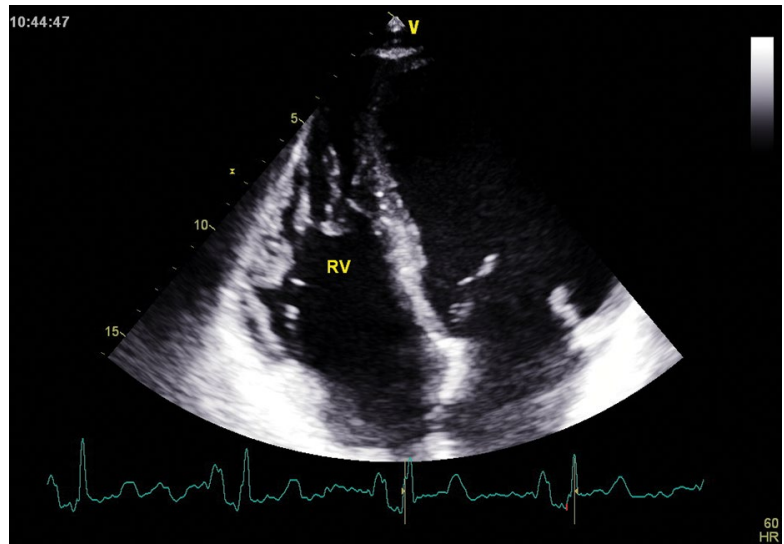

Figure 2. The four-chamber view - right ventricular free wall affected by non-compaction

depicted with a two-layered pattern of the wall, consisting of a C epicardial layer and NC endocardial layer, typical of LVNC. In the end-systolic phase, the NC:C ratio in the parasternal short axis view was approximately 3 (Figure 1). The Color Doppler imaging showed blood flow from the left ventricular lumen into the sinuses of the NC layer. The above-described imaging results met the diagnostic Jenni's [3] criteria for LVNC. The entire free wall of the right ventricle was also affected by non-compaction (Figure 2). There were no significant abnormalities found in TTE, excluding mild mitral regurgitation and trace aortic regurgitation. The whole clinical presentation enabled making the diagnosis of NCC of both ventricles. Coronary angiography found normal epicardial coronary arteries, thus excluding a coronary background of myocardial injury. The diagnosis was extended to include an 24-hour ECG Holter monitoring, which detected several episodes of non-sustained ventricular tachycardia (Figure 3). It was concluded with high probability that the loss of consciousness was cardiogenic in nature and it was associated with hazardous ventricular arrhythmia. Accordingly, the patient was implanted with an implantable cardioverter-defibrillator (ICD) in the secondary prevention of sudden cardiac death. Then, a cardioverter-defibrillator test was performed under brief general anaesthesia, which confirmed proper functioning of the device. Before the patient was discharged from the hospital, an echocardiographic follow-up was performed. The said examination detected the presence of previously absent thrombus in the left ventricular apex. The presence of thrombus was confirmed by tissue Doppler imaging as well as contrast-enhanced ultrasound (Figure 4).

The therapy included bisoprolol, trandolapril, spironolactone, torasemide and unfractionated heparin used in continuous infusions, followed by vitamin $\mathrm{K}$ antagonist. A gradual regression of the thrombus was observed in 


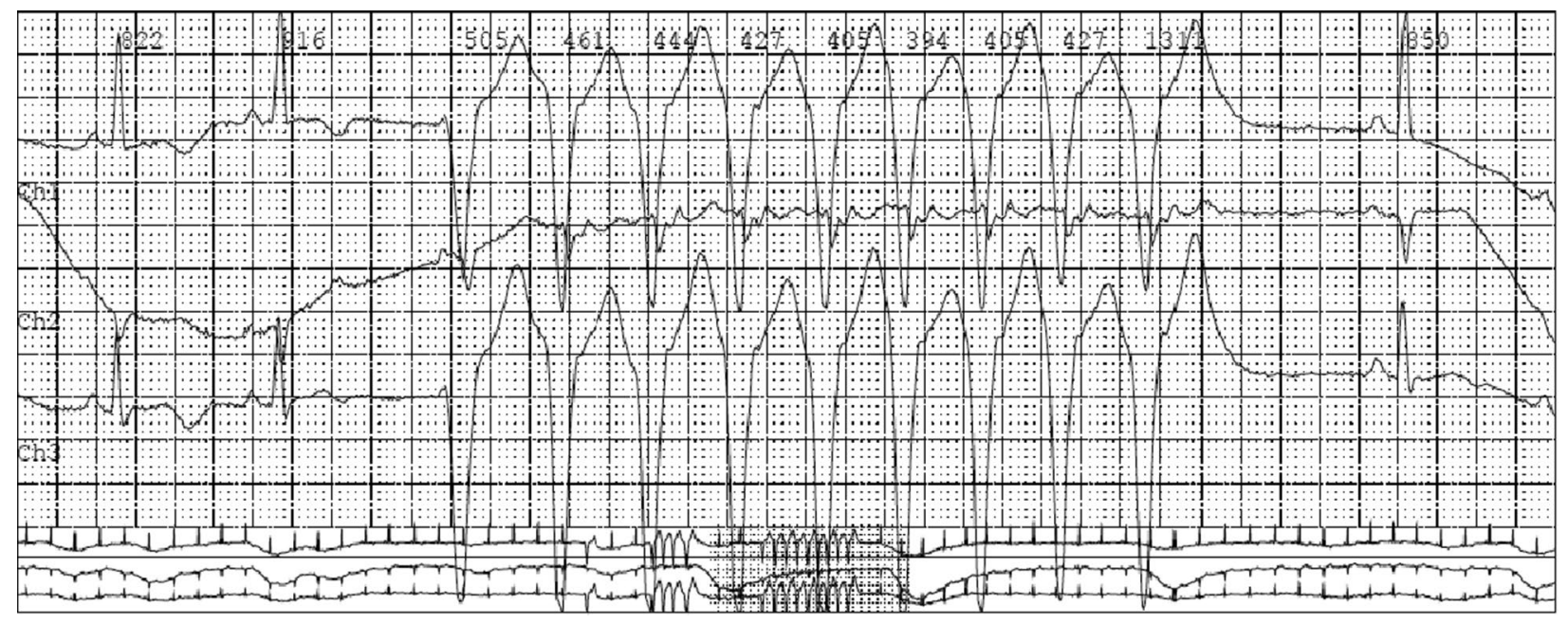

Figure 3. A non-sustained ventricular tachycardia episode detected on Holter trace

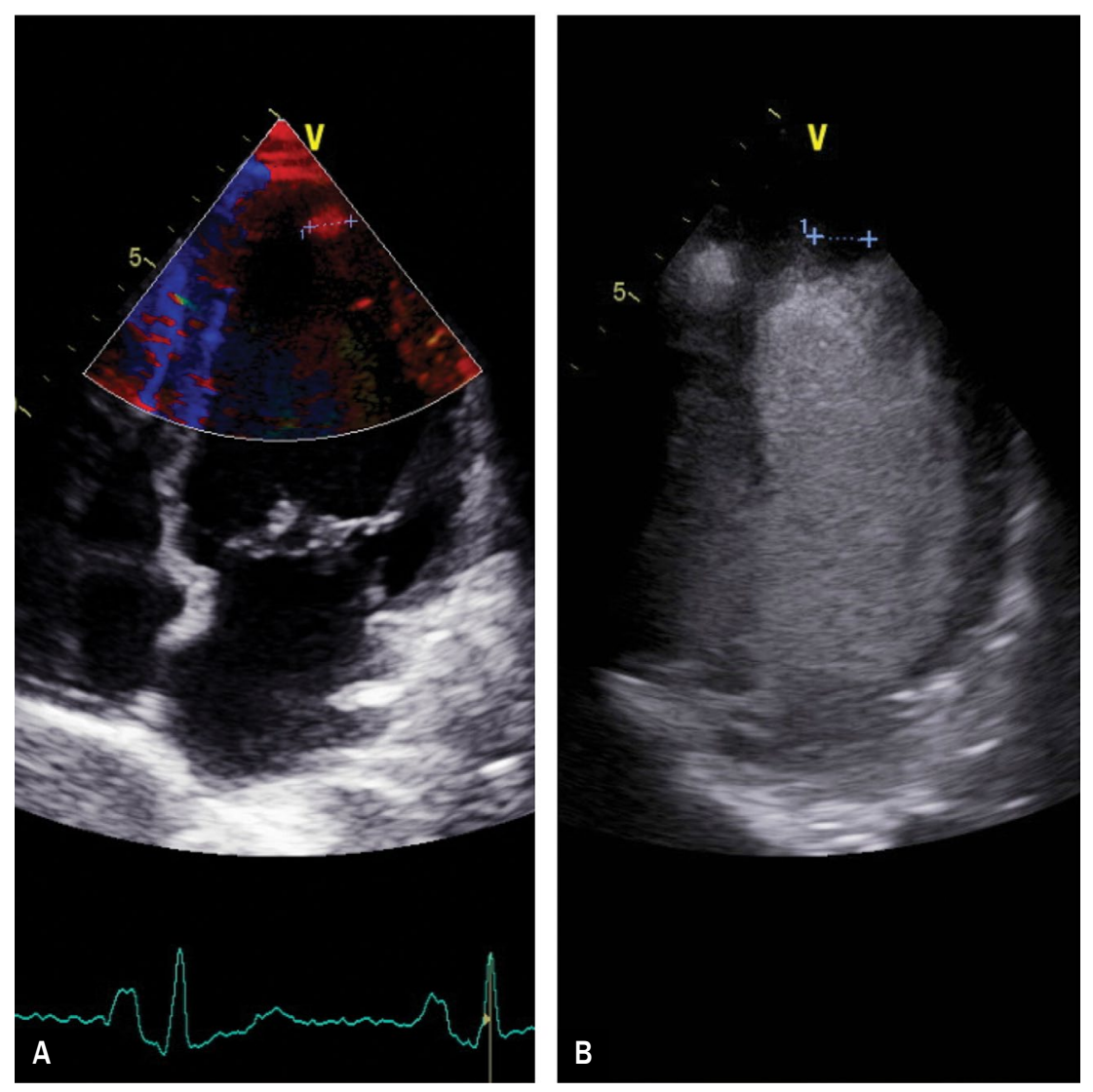

Figure 4A, B. The four-chamber view, tissue Doppler imaging and contrast-enhanced ultrasound - left ventricular apical thrombus

echocardiographic follow-ups. The patient was discharged home in good condition with a recommendation to continue treatment in an outpatient setting. Two weeks after the patient's hospital discharge, an outpatient visit revealed his overall good condition, no ICD interventions. Echocardiography excluded the left ventricular apical thrombus.

\section{Summary}

The above-mentioned case study presents a patient without overt clinical signs of heart failure, which are the most common manifestation in a group of patients with NCC. The first manifestation of NCC was brief loss 
of consciousness, the cause of which was considered to be hazardous ventricular arrhythmia. The overall clinical picture suggested the need for implantation of a cardioverter-defibrillator in the secondary prevention of sudden cardiac death. The high risk of thromboembolic complications in this group of patients is emphasised by the fact that the duration of hospitalisation was sufficient enough for the development of left ventricular apical thrombus in the patient.

The absence of typical signs of heart failure does not exclude significant myocardial damage. Early diagnosis, treatment and follow-up of this group of patients improve prognosis and prevent sudden cardiac death early enough. In the case of patients with confirmed myocardial non-compaction, despite the absence of signs or dysfunctions of the left ventricle, echocardiographic screening of family members is recommended to exclude this pathology.

\section{References}

1. Pietraszek N, Piotrowska-Kownacka D, Główczyńska R. Kardiomiopatia z niescalenia mięśnia lewej komory. Folia Cardiol. 2018; 13(4): 378-385, doi: 10.5603/fc.2018.0081.

2. Ponikowski P, Hoffman P, Witkowski A, Lipiec P. ed. Kardiologia. Podręcznik Polskiego Towarzystwa Kardiologicznego. Via Medica, Gdańsk 2019: 487-488.

3. Jenni R, Oechslin E, Schneider J, et al. Echocardiographic and pathoanatomical characteristics of isolated left ventricular non-compaction: a step towards classification as a distinct cardiomyopathy. Heart. 2001; 86(6): 666-671, doi: 10.1136/heart.86.6.666, indexed in Pubmed: 11711464. 